\title{
Resistance in Candida albicans: Exploring the Cell Wall Barrier by Proteomics
}

Bruno Maras ${ }^{1}$, Giuseppina Mignogna ${ }^{1}$ and Letizia Angiolella ${ }^{2 *}$

${ }^{1}$ Department of Biochemical Sciences "A. Rossi Fanelli ", Sapienza University of Rome, Italy

${ }^{2}$ Department of Public Health and Infectious Diseases. Sapienza University of Rome, Italy

*Corresponding author: Letizia Angiolella, Department of Public Health and Infectious Diseases, University of Rome, " Sapienza", Piazzale Aldo Moro, 00161-Rome, Italy, Tel: +3964468625; E-mail: letizia.angiolella@uniroma1.it

Received date: 08 July, 2015; Accepted date: 20 September, 2015; Published date: 25 September, 2015

Copyright: $\odot 2015$ Maras B, et al. This is an open-access article distributed under the terms of the Creative Commons Attribution License, which permits unrestricted use, distribution, and reproduction in any medium, provided the original author and source are credited.

\begin{abstract}
The incidence of candidiasis has dramatically increased and bloodstream infections due to different species of Candida are becoming a prime cause of morbidity and mortality in different types of immunocompromised patients. Azole and echinocandin drug resistance accounts for the dramatic increase in incidence of nosocomial bloodstream candidiasis found in recent years. Cell wall constitutes the barrier between the yeast and the host and resistant strains change the proteome of this compartment. In the last decade different proteomic platforms have been applied to study cell wall and markers of resistance to drugs have been pointed out. Modulation of these proteins seem to suggest that although resistance is based on a specific mutation able to counteract the toxicity of the antifungal drug, a set of other molecular modifications takes place contemporary or subsequently the establishment of the resistance and seems to support the viability of the resistant yeast. Profiled proteins by proteomics may be valuable in design therapy using classical antifungal along with complementary drugs able to abolish pathways that strengthen the resistance and attenuate virulence of the mutated cell.
\end{abstract}

\section{Key words:}

Cell wall; Candida albicans; Proteomics; Drug resistance

\section{Introduction}

Candida albicans, a dimorphic opportunistic human pathogen yeast, is the most prominent cause of oro-pharyngeal, vaginal and invasive candidiasis in man [1]. In particular, oro-pharyngeal infections are very common in human immunodeficiency virus (HIV)infected individuals and patients with acquired immune deficiency syndrome (AIDS), while deep-seated infections are frequent in neutropenic patients under chemotherapy $[2,3]$.

Currently available therapies reside on antifungal drugs belonging to azole and echinocandin families that interfere with different aspects of fungal metabolism.

In Candida, azole antifungals target protein Erg11p involved in the biosynthesis of ergosterol, the main sterol of the membranes of the fungal cell. This protein is located at the inner face of the membrane of the smooth endoplasmic reticulum, engaged in the synthesis of lipids, including oils, phospholipids and steroids. Erg11p, a member of the cytochrome P450 family, is a hemoprotein that has an iron atom as prosthetic group, whose function is to fix the dioxygen necessary for the enzymatic activity [4]. Echinocandin drugs are lipopeptides that inhibit glucan synthase, which is responsible for the biosynthesis of B-1,3-D-glucan, a major structural component of fungal cell wall [5]. The echinocandin target, $B-1,3-D$-glucan synthase, is a fungal-specific multi-subunit enzyme complex comprised of Rho, a GTP-binding protein, which helps regulate the overall activity of glucan synthase and a catalytic subunit Fks encoded by three related genes, FKS1, FKS2, and FKS3 [6].

\section{Candida resistance to antifungal drugs}

The raise of resistant strains to these drugs account for the dramatic increase in incidence of nosocomial bloodstream candidiasis found in recent years [7]. Acquired resistance in Candida has been described and underlying mechanisms have been characterized for both azoles and echinocandins. For the azoles, three different mechanisms have been described: 1) mutations of ERG11 that reduce the binding affinity of the protein to azole antifungals. These mutations are not randomly distributed over the ERG11 gene but are preferentially clustered within three regions [4] ; 2) overexpression of ERG11, leading to an increase of the intracellular concentration of the target protein. Two independent mechanisms drive ERG11 overexpression: the former is ERG11 gene duplication while the latter depends on the regulation of the ergosterol biosynthesis pathway; 3) overexpression of efflux membrane transporters for azole drug that carrying azole outside the cell decreases their intracellular concentration. Two families of drug efflux transporters can be distinguished in yeasts [4]: the ATP Binding cassette $(\mathrm{ABC})$ transporters, encoded by the genes CDR1 and CDR2 in Candida albicans and the major facilitator superfamily (MFS) transporter encoded by MDR1 gene [8].

In the individual isolate these mechanisms often act in concert leading to stepwise increases in minimal inhibition concentration (MIC) and broadening of the azole resistance spectrum.

For the echinocandins only target gene mutations of $B-1,3-\mathrm{D}$-glucan synthase are responsable for resistance in isolates and produce modification of the catalytic Fsk subunit (Fks1 and Fks2) of glucan synthase [9]. Unlike azole antifungal agents, echinocandins are not substrates for multidrug transporters [10]. Echinocandin resistance is conferred by characteristic amino acid substitutions in Fks subunits [11], which induce elevated MIC values (10-100 fold) and reduce the sensitivity of glucan synthase (IC50) to drug by 50 - to 3,000 -fold [12]. 
Characteristic mutations in FKS genes are prominently associated with reduced clinical response [13].

\section{Molecular studies of Candida cell wall}

To better understand at molecular level the organization of the fungal cell once drug resistance has been established, cell wall represents a first choice as this compartment constitutes her barrier between the yeast the and host [14]. This essential organelle maintains cell shape strengthening its structure, limits permeability thereby retaining periplasmic proteins and protects from host degrading enzymes. The cell wall is mainly composed of beta-1,3- and beta-1,6glucans and by small amount of chitin long with proteins that can be grouped in three main classes: i) glycophosphatidylinositol (GPI)anchored proteins(GPI-CWPs), the majority of them mannosylated, and covalently linked to the beta-1,6-glucan through the GPI anchor; ii)proteins with Internal Repeats (PIR) directly linked to the beta-1,3glucan iii) CWPs lacking a covalent attachment to the polysaccharide matrix $[11,15]$.

Studies for identification of CWPs that change their expression in resistance strains may be useful to determine biological markers associate to drug resistance with a diagnostic value and to find out antigenic epitopes that can be used to evoke antibodies able to contain Candida invasion. Furthermore, determination of the functional properties of differently expressed CWP may highlight metabolic pathways involved in sustaining the virulence of the yeast. A suitable strategy to study modulation of cell wall proteins in experimental resistance strains and clinical isolates is represented by the proteomic approach that can yield a complete comparative picture of the differential expressed proteins between sensitive and resistant strains.

Different proteomic platforms have been applied to study different topics of Candida albicans as reported in 137 published in PubMed in the last decade (crossing terms: proteomics and Candida albicans). 32 out of these 137 papers, are indeed focused on cell wall structure $[16,17]$, metabolism [18], surface immunogenic proteins and proteins enriched in the cell wall [19] and conveyed outside in extracellular vesicles [20]. The limited number of articles, despite the significance and importance of this compartment in Candida metabolism, may reside on the difficulty of preparation and purification of the cell wall proteome since most of the proteins, GPI-CWP and PIR are covalently linked to the glucan moiety. In particular scant information are still available on changes of cell wall proteoma in antifungal resistant strains.

Thanks to a 2D-electrophoretic based proteomic platform, two proteins, bgl2p [21] and rhd3/pga29 [22], whose expression clearly increase on the cell wall in micafungin resistant strains have been recently identified and up to now they represent the main cell wall markers in the resistance to this antifungal drug. Bgl2p is an endo-1,3glucanase and glucanosyltransferase that introduces 1,6-linkages in 1,3 -glucan chains and participates in cross-linking of the glucan component of the cell wall. Bgl2p has also been found in exosomes released from Candida albicans [20]. Deletion of BGL2 does not obviously affect cell morphology, whereas its over-expression is harmful for cell viability. It is noteworthy that an increase of bgl2p has been observed also in fluconazole resistant strains indicating a beneficial role of this protein probably to favour the re-arrangment of cell wall in both resistant strains. Antibodies against this protein represent an accurate diagnostic biomarkers in patients with systemic candidiasis along with anti-wall phosphoglicerate kinase antibodies and high seropositivity to anti-bgl2p modulate anti-wall enolase response lowering risk of fatality in these patients [22].

Rhd3/pga29, the latter biomarker of micafungin resistance, has been recently identified by us [23]. For this protein, a functional role has not yet been assigned. However, deletion of gene attenuated the pathogenicity and diminished the production of pro-inflammatory cytokines by the host indicating a peculiar role of this protein in the virulence of the fungus [24]. An up-regulation of this protein occurs in presence of iron while it is down-regulated during the yeast to hyphae transition. Rhd3/pga29 increase is peculiar of the resistance to micafungin since in fluconazole resistant strains there is not any increase of this marker on the cell wall. This different response indicates aspecific molecular adaptation regarding Rhd3/pga29 levels on the cell wall of resistant cells to the two antifungal drugs.

Modulation of these proteins seem to suggest that although resistance is based on a specific mutation able to counteract the toxicity of the antifungal drug, a set of other molecular modifications takes place contemporary or subsequently the establishment of the resistance and seems to support the viability of the resistant yeast cell. More sensitive and quantitative proteomic approaches will be helpful in widening the list of proteins involved in the re-organization of the cell in echinocandins and azoles resistance. Identification of these proteins may help in defining the molecular processes involved in resistant strains fitness. These new technologies could be applied also to identify down-regulated protein that, if antigenic, could allow the fungus to escape immunogenic response.

\section{Conclusion}

Profiled proteins modulated in resistant strains may be valuable in design therapy using classical antifungal along with complementary drugs able to abolish pathways that strengthen the resistance and attenuate virulence of the mutated cell. This strategy may succeed in overcoming Candida albicans invasion mainly in immunocompromised patients where resistant strains to both azoles and echinocandins frequently occur.

\section{References}

1. Calderone RA, Clancy CJ (2012) Candida and candidiasis. (2nd edn.) ASM Press, Washington, DC

2. Fidel PL Jr (2011) Candida-host interactions in HIV disease: implications for oropharyngeal candidiasis. Adv Dent Res 23: 45-49.

3. Eggimann P, Pittet D (2014) Candida colonization index and subsequent infection in critically ill surgical patients: 20 years later. Intensive Care Med 40: 1429-1448.

4. Noël T (2012) The cellular and molecular defense mechanisms of the Candida yeasts against azole antifungal drugs. J Mycol Med 22: 173-178.

5. Onishi J, Meinz M, Thompson J, Curotto J, Dreikorn S, et al. (2000) Discovery of novel antifungal $(1,3)$-beta-D-glucan synthase inhibitors. Antimicrob Agents Chemother 44: 368-377.

6. Perlin DS (2014) Echinocandin resistance, susceptibility testing and prophylaxis: implications for patient management. Drugs 74: 1573-1585.

7. Bondaryk M, kurzatkowski W, Staniszewska M (2013) Antifungal agents commonly used in the superficial and mucosal candidiasis treatment: mode of action and resistance development. Postepy Dermatol Alergol 30: 293-301.

8. Prasad R, Rawal MK (2014) Efflux pump proteins in antifungal resistance. Front Pharmacol 5: 202.

9. Yamaguchi H (1999) Molecular and biochemical mechanisms of drug resistance in fungi. Nihon Ishinkin Gakkai Zasshi 40: 199-208. 
Citation: Maras B, Mignogna G, Angiolella L (2015) Resistance in Candida albicans: Exploring the Cell Wall Barrier by Proteomics. Chemo Open Access 4: 165. doi:10.4172/2167-7700.1000165

Page 3 of 3

10. Niimi K, Maki K, Ikeda F, Holmes AR, Lamping E, et al. (2006) Overexpression of Candida albicans CDR1, CDR2, or MDR1 does not produce significant changes in echinocandin susceptibility. Antimicrob Agents Chemother 50: 1148-1155

11. Klis FM, de Groot P, Hellingwerf K (2001) Molecular organization of the cell wall of Candida albicans. Med Mycol 39 Suppl 1: 1-8.

12. Garcia-Effron G, Lee S, Park S, Cleary JD, Perlin DS (2009) Effect of Candida glabrata FKS1 and FKS2 mutations on echinocandin sensitivity and kinetics of 1,3-beta-D-glucan synthase: implication for the existing susceptibility breakpoint. Antimicrob Agents Chemother 53: 3690-3699.

13. Lackner M, Tscherner M, Schaller M, Kuchler K, Mair C, et al. (2014) Positions and numbers of FKS mutations in Candida albicans selectively influence in vitro and in vivo susceptibilities to echinocandin treatment. Antimicrob Agents Chemother 58: 3626-3635.

14. Heilmann CJ, Sorgo AG, Klis FM (2012) News from the fungal front: wall proteome dynamics and host-pathogen interplay. PLoS Pathog 8: e1003050.

15. Chaffin WL (2008) Candida albicans cell wall proteins. Microbiol Mol Biol Rev 72: 495-544.

16. Klis FM, de Koster CG, Brul S (2011) A mass spectrometric view of the fungal wall proteome. Future Microbiol 6: 941-951.

17. Castillo L, Calvo E, Martínez AI, Ruiz-Herrera J, Valentín E, et al. (2008) A study of the Candida albicans cell wall proteome. Proteomics 8: 3871-3881.
18. Thomas DP, Pitarch A, Monteoliva L, Gil C, Lopez-Ribot JL (2006) Proteomics to study Candida albicans biology and pathogenicity. Infect Disord Drug Targets 6: 335-341.

19. Sáez-Rosón A, Sevilla MJ, Moragues MD (2014) Identification of superficial Candida albicans germ tube antigens in a rabbit model of disseminated candidiasis. A proteomic approach. Int Microbiol 17: 21-29.

20. Gil-Bona A, Llama-Palacios A, Parra CM, Vivanco F, Nombela C, et al. (2015) Proteomics unravels extracellular vesicles as carriers of classical cytoplasmic proteins in Candida albicans. J Proteome Res 14: 142-153.

21. Angiolella L, Vitali A, Stringaro A, Mignogna G, Maras B, et al. (2009) Localisation of Bgl2p upon antifungal drug treatment in Candida albicans. Int J Antimicrob Agents 33: 143-148.

22. Pitarch A, Jiménez A, Nombela C, Gil C (2006) Decoding serological response to Candida cell wall immunome into novel diagnostic, prognostic, and therapeutic candidates for systemic candidiasis by proteomic and bioinformatic analyses. Mol Cell Proteomics 5: 79-96.

23. Vavala E, Mignogna G, Spano F, Stringaro A, Colone M, et al. (2013) The cell wall protein Rhd3/Pga29 is over-expressed in Candida albicans upon micafungin treatment. J Chemother 25: 332-340.

24. de Boer AD, de Groot PW, Weindl G, Schaller M, Riedel D, et al. (2010) The Candida albicans cell wall protein Rhd3/Pga29 is abundant in the yeast form and contributes to virulence. Yeast 27: 611-624. 